\title{
Fair Task Allocation in Crowdsourced Delivery
}

\author{
Fuat Basık $^{\dagger *}$, Buğra Gedik ${ }^{\dagger}$, Hakan Ferhatosmanoglu ${ }^{\S}$, Kun-Lung Wu $\ddagger$ \\ † Department of Computer Engineering, Bilkent University, Turkey \\ $\S$ Department of Computer Science, University of Warwick, UK $\ddagger$ IBM Research, New York, USA \\ fuat.basik@bilkent.edu.tr, bgedik@cs.bilkent.edu.tr, hakan.f@warwick.ac.uk, klwu@us.ibm.com
}

\begin{abstract}
Faster and more cost-efficient, crowdsourced delivery is needed to meet the growing customer demands of many industries, including online shopping, on-demand local delivery, and on-demand transportation. The power of crowdsourced delivery stems from the large number of workers potentially available to provide services and reduce costs. It has been shown in social psychology literature that fairness is key to ensuring high worker participation. However, existing assignment solutions fall short on modeling the dynamic fairness metric. In this work, we introduce a new assignment strategy for crowdsourced delivery tasks. This strategy takes fairness towards workers into consideration, while maximizing the task allocation ratio. Since redundant assignments are not possible in delivery tasks, we first introduce a 2-phase allocation model that increases the reliability of a worker to complete a given task. To realize the effectiveness of our model in practice, we present both offline and online versions of our proposed algorithm called F-Aware. Given a task-to-worker bipartite graph, F-Aware assigns each task to a worker that minimizes unfairness, while allocating tasks to use worker capacities as much as possible. We present an evaluation of our algorithms with respect to running time, task allocation ratio (TAR), as well as unfairness and assignment ratio. Experiments show that F-Aware runs around $10^{7} \times$ faster than the TAR-optimal solution and allocates $96.9 \%$ of the tasks that can be allocated by it. Moreover, it is shown that, F-Aware is able to provide a much fair distribution of tasks to workers than the best competitor algorithm.
\end{abstract}

Index Terms-spatial crowdsourcing, crowdsourced delivery, fairness.

\section{INTRODUCTION}

Spatial crowdsourcing has emerged as a viable solution for delivery logistics, such as on-demand local delivery, online shopping, and on-demand transportation [10]. As such, it has attracted significant attention from both the academia and the industry in recent years. For instance, Amazon utilizes the crowd to provide same day shipment of packages from warehouses to customers 1 Postmates ${ }^{2}$, a company offering on demand food and delivery, is available all around the US. Enormous growth of the crowdsourced taxi services Uber ${ }^{3}$ and Lyf $\mathrm{I}^{4}$ has attracted significant interest, resulting in numerous research studies being conducted about them.

Crowdsourced delivery applications have three stakeholders: customers, workers and the matching platform. Customers submit tasks of spatial deliveries to the platform. The platform matches the tasks with the workers' availabilities, and allocates workers to tasks considering the spatio-temporal requirements. To support faster and cheaper delivery, spatial crowdsourcing platforms require a critical mass of workers. The workers should be attracted by a high income potential which is possible with a large number of customers. This situation drives these platforms into a chicken and egg problem [29], in which a powerful network is necessary to attract customers and customers are necessary to engage a powerful network.

\footnotetext{
* Part of this work was done while the author was an intern at IBM.

1. http:/ / flex.amazon.com

2. http://www.postmates.com

3. http://www.uber.com

4. http://www.lyft.com
}

A negative correlation between job satisfaction and worker turnover is naturally expected in crowdsourcing environments. According to a study with MTurk workers, a common indicator of positive behavior of the employer, hence the job satisfaction, is fairness [7]. Fairness can be defined in the context of anti discrimination laws, equity of opportunity and equality of outcome [37], [3]. In the context of crowdsourcing, the distributive fairness is particularly relevant [16], [17]. This definition seeks fairness based on the proximity between a worker's own input/output ratio and the input/output ratio of a referent [1]. For example, the workers would expect to be assigned a fair number of tasks that is proportional to their spatio-temporal matching qualities/availabilities for the tasks. Effect of such fairness expectations on the likelihood of participation is more than that of considerations of self-interest [17]. Hence, fairness needs to be considered as an essential concept for sustaining a powerful crowd with significant participation of workers [5].

In this paper, we study the problem of fair allocation of delivery tasks to workers within the context of spatial crowdsourcing. The tasks are associated with receive and delivery locations and time constraints. The workers inform the platform about their working status using availabilities, i.e., the location and time period they are willing to serve. While the primary objective is to maximize the task allocation ratio (TAR), which is the ratio of number of allocated tasks to number of all tasks, we aim to achieve this via a fair distribution of tasks to workers. Current approaches focus only on the first objective of maximizing the number of tasks under certain constraints from workers [21]. While this reduces the use of third party services or employing 
full time couriers [29], it ignores fairness and worker satisfaction. This can result in lower engagement and migration to other services. [16].

There is a number of challenges to achieve an effective and fair crowdsourced delivery. First, unlike other crowdsourcing applications, a redundant allocation of tasks is not possible in the crowdsourced delivery. Redundant allocation improves the reliability of task completion by increasing the number of workers the task is assigned to [10]|21]. In delivery tasks, however, only one worker can be allocated to complete the task. Hence, to increase the reliability of the worker selection in such tasks, we employ a 2-phase allocation model. In the first phase, the platform selects a set of nominees among available workers and the task is offered to a subset of them. In the second phase, the platform selects one worker among those who accepted the offer. This avoids the broadcasting of the offer to all nominees in the first phase to forestall spamming of the workers.

The second challenge is to provide distributional fairness among workers to ensure participation. Unless workers and their availabilities are identical, assigning each worker an equal number of tasks is not a fair distribution, as it does not take the user input into consideration. To address this issue, we call the input/output ratio local assignment ratio and set the output of a worker as the amount of revenue she gets from the system, while the input is the total reward of the offers she has accepted (not necessarily allocated). This view allows us to define a technical measure of the global fairness as the coefficient of variation, a statistical measure of relative variability, of all local assignment ratios. A low coefficient of variation is associated with the fairness of allocation.

Without considering fairness, the task allocation problem can be reduced to the minimum cost flow (MCF) problem [21]. However, the MCF-based solutions fall short to capture fairness, since every assignment needs to update its cost matrix. We introduce F-Aware as a solution to assign tasks to workers in a bipartite graph, by minimizing the unfairness locally and allocating tasks to fill worker capacities.

The third challenge is to handle online allocation. In applications such as online shopping (e.g., with a 3-hour delivery guarantee), the platform can allocate multiple tasks in mini-batches with no global knowledge of all tasks and availabilities in advance. In contrast, in on-demand transportation services, like Uber and Lyft, customers want to know whether the vehicle is on the way, almost instantly. Therefore the allocation should be done at the very moment of the task arrival. F-Aware with the 2-phase model is shown to be applicable for offline, online, and mini-batch allocation strategies.

In summary, this paper makes the following contributions:

- Model. We introduce a generic task allocation model to cover a variety of crowdsourced delivery scenarios. The 2-phase allocation model increases the reliability of task completion by double-checking a worker's willingness to complete the tasks. This model handles the case where a potential worker may refuse the task even though she is available.

- Algorithm. We introduce a fairness-aware solution called F-Aware, which locally minimizes unfairness, while targeting maximum task allocation. The MCFbased algorithms fall short on modeling the dynamic fairness metric, and are not feasible for the online scenarios.

- Online Allocation. We enhance our 2-phase model to perform online task allocation. We show that $F$ Aware is effective also for online and mini-batch allocation scenarios, as it is for offline allocation.

We provide a comprehensive experimental study using real-world datasets to showcase the effectiveness and efficiency of our 2-phase model and of the F-Aware algorithm in terms of running time, task allocation ratio, and fairness it achieves.

The rest of this paper is organized as follows. Section 2 gives the preliminaries of the problem. Section 3 explains the details of our 2-phase task allocation model. Section 4 extends our approach to online task allocation. Section 5 presents the experimental evaluation. Section 6 discusses the related work. Finally, Section 7 concludes the paper.

\section{Problem Definition}

We aim to develop a new strategy on allocation of tasks to workers in a crowdsourced environment. The overall goal is to maximize the task allocation ratio (TAR), the ratio of number of allocated task over the number of all tasks, while distributing tasks to workers fairly. We now give the preliminaries of the domain and formalize this multi-criteria optimization problem.

Definition 1. Time period. A time period, $h$, is a pair of datetime values $b$ and $e$, representing the beginning and end times, respectively.

Definition 2. Delivery task. Tasks are in the form of spatiotemporal deliveries, such as workers need to move to the source of the delivery to receive the item and deliver it to the recipient. In this manner, one can consider a task as a composite of receive and deliver steps. We represent the set of all tasks with $\mathcal{T}$, and $i^{\text {th }}$ task with $t_{i}$. A task is a quintuple $\left\{h_{s}, l_{s}, h_{r}, l_{r}, m\right\}$. Here, $h$ and $l$ represents a time period and a geo-spatial point such as a latitude/longitude pair, respectively. Subscripts $s$ and $r$ stands for the source and the destination of the task. In other words, $t_{i} \cdot l_{s}$ stands for location of the source while $t_{i} . l_{r}$ stands for location to deliver the item for $i^{t h}$ task $t_{i}$. Note that these steps are associated with different time periods, as receive and deliver steps have their own validity periods $h_{s}$ and $h_{r}$. Lastly, $m$ represents the reward of the task.

Definition 3. Worker. Workers are people who participate in the platform to make money. We represent the set of all workers with $\mathcal{W}$, and $i^{\text {th }}$ worker with $w_{i}$. Each worker, is a triple $\{A, c, f\} . w_{i} . A$ is the set of availabilities of worker $w_{i}, w_{i} . c$ is her capacity and $w_{i} . f$ is the local assignment ratio. Local assignment ratio, which will be detailed later, is a dynamic metric used to determine how fair the system treated a worker so far, defined as the ratio of the worker's revenue over the total reward of the offers she has accepted. The revenue of the worker equals to sum of rewards of the tasks allocated to her. 
Customers

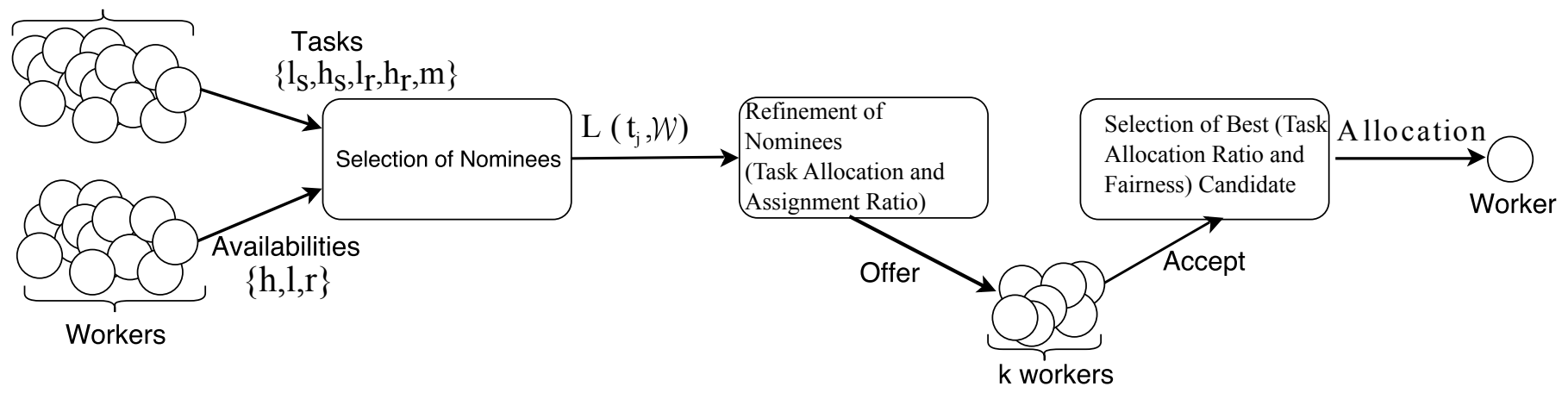

Fig. 1: Tasks and availabilities are the inputs of the platform. For each task, the nominees are identified. During this calculation, the system also finds out the acceptance probability, based on Equation 8 . The task is multicasted to $k$ of those nominees. Workers who accept the offer are referred to as candidates and one worker among the candidates is selected for allocation.

Definition 4. Availability. Workers inform the system about their working status using availabilities. $a_{i p}$ is the $p^{\text {th }}$ availability of $i^{t h}$ worker $w_{i}$, such that $a_{i p} \in w_{i}$.A. Each availability is a triple, $\{h, l, r\} . a_{i p} . l$ is a geo-spatial point. It is the center of the region in which $w_{i}$ is willing to accept tasks. $a_{i p} . r$ is the radius of the same region. Let us represent this region with the $C r(l, r)$ function. The worker is ready to serve during the time period $a_{i p} . h$.

For a task, $t_{i}$, to be completed, a worker has to move to the receive location, $t_{i} . l_{s}$, during its validity period $t_{i} . h_{s}$; and after that, she has to move to the deliver location, $t_{i} . l_{r}$, during its validity period $t_{i} \cdot h_{r}$. An example task $t_{i}$ in crowdsourced delivery could be to pick-up a gift item from a local shop $\left(t_{i} . l_{s}\right)$ between 12:00 and 14:00 on 23rd of December 2016 $\left(t_{i} . h_{s}\right)$, and deliver it to a home address $\left(t_{i} . l_{r}\right)$ between 18:00 and 22:00 on the same day $\left(t_{i} . h_{r}\right)$. In return for completing this task, the worker will be paid $t_{i} . m$ amount of money. In the real world, multiple parameters effect the $t_{i} . m$ value, including the distance between $t_{i} \cdot l_{s}$ and $t_{i} \cdot l_{r}$, the size of the package, and the sensitivity of the content, etc.

\subsection{Fairness}

An effective network is key to building a powerful crowdsourcing platform, therefore, providing continuous participation of workers and avoiding worker turnover are crucial. A negative correlation is naturally expected between job satisfaction and worker turnover in crowdsourcing environments. According to a study with MTurk 5 workers, 11 to 26 percent of turnover in crowdsourcing environment is explained with job satisfaction. In the same study, fairness is listed as one of the most common indicator of the job satisfaction [7], [30]. Fairness needs to be considered as a first class citizen in designing crowdsourcing applications to ensure long term commitment and participation [5].

There are three major forms of fairness defined in the social psychology literature, namely: procedural, interactional, and distributive. Procedural fairness is the perception of justice on the procedures, policies, and the criteria used by the decision maker [16]. Interactional fairness is the interpersonal aspect of the procedural fairness. Distributive fairness

5. http://www.mturk.com/ is defined as the proximity between a worker's own input/output ratio and the input/output ratio of a referent [1]. Prior research on the relationship between fairness and job satisfaction shows that when fairness is regressed along all three dimensions, the job satisfaction gets impacted the most due to the loss of distributed fairness [30]. Note that, unless the workers are identical, assigning each worker to an equal number of tasks is not a fair distribution by this definition, as it does not take user input into consideration. Therefore, we define a new fairness model that captures distributive fairness, which will be detailed shortly.

\subsection{Formalization}

With the given definitions, let us first define the problem before discussing each component separately.

Fair allocation of delivery tasks in a crowdsourcing environment: Given the set of delivery tasks $\mathcal{T}$ and the set of workers $\mathcal{W}$, represented with their availabilities, the problem is allocating tasks to workers with the goals of maximizing the task allocation ratio $(T A R)$ and minimizing the unfairness $(\mathcal{F})$ objectives, under the candidacy, capacity and assignment ratio (AR) constraints which will be explained next.

Task Allocation Ratio (TAR): To reduce the dependency of the businesses to using a third party service we set maximizing task allocation ratio (TAR) as the first component of our objective function. $T A R$ is defined as the ratio of the number of allocated tasks over the number of all tasks. Formally, let $T_{\text {all }}$ be the set of allocated tasks and $\mathcal{T}$ be the set of all tasks. The $T A R$, defined as:

$$
T A R=\frac{\left|T_{\text {all }}\right|}{|\mathcal{T}|}
$$

Unlike other crowdsourcing applications, redundant allocation of tasks is not possible in crowdsourced delivery. Therefore, in order to increase the reliability of a worker completing a given task, the allocation is done via a 2-phase procedure, illustrated in Figure 11. In the first phase, the system nominates a set of workers whose availabilities are suitable to complete the task. The task is offered to these 
nominees, and they have an opportunity to accept or reject it. A worker may refuse the offer, even though she is available. For brevity, we leave the details of nomination and offering strategy to the next section. Workers who have accepted the offer are called candidates. In the second phase, one worker among the candidates is selected and is assigned to the task.

Fairness: Recall that distributive fairness is defined as the proximity between a worker's own input/output ratio and the input/output ratio of a referent. In the 2-phase allocation model, the input of a worker is the total reward of the offers she accepted. Note that availabilities cannot be used as input since a worker might reject offers even though she is available. The output of a worker, on the other hand, is the amount of money she earned. Intuitively, not all tasks have the same complexity, yet the reward of each task is proportional to its hardness. To capture the hardness of the tasks while determining a worker's input/output ratio, instead of counting the number of tasks a worker accepted or got assigned, we use the reward of each task. Each worker $w_{i}$ is associated with a local assignment ratio (LAR), w. $f_{i}$, defined as the ratio of the total reward of tasks allocated to a worker (output) over the total reward of tasks she has accepted (input). Formally, let $w_{i} \cdot T_{\text {all }}$ be the set of tasks allocated to worker $w_{i}$, and $w_{i} \cdot T_{a c c}$ is the set of offers she has accepted, the LAR of $w_{i}$ is defined as:

$$
w_{i} . f=\frac{\sum_{j=1}^{x} t_{j} \cdot m}{\sum_{k=1}^{y} t_{k} \cdot m}, \forall_{j, k} t_{j} \in w_{i} \cdot T_{a l l}, t_{k} \in w_{i} \cdot T_{a c c}
$$

where

$$
x=\left|w_{i} . T_{a l l}\right| \text { and } y=\min \left(\left|w_{i} \cdot T_{a c c}\right|, w_{i} . c\right)
$$

The number of tasks a worker accepts can exceed her capacity, since she will be assigned to a subset of the tasks she has accepted. However, since the capacity limits the number of allocations, we consider minimum of $\left\{\left|w_{i} . T_{a c c}\right|, w_{i} . c\right\}$ acceptances when calculating the denominator. The system is considered more fair as the proximity of the $L A R$ values will be higher. Although the standard deviation of the set of $L A R$ values represents this proximity, using it as the evaluation metric would be misleading since the different allocation schemes will have different number of tasks allocated. Hence, we evaluate the overall fairness of the system, $\mathcal{F}$, using coefficient of variation of the set of $L A R$ values, i.e., standard deviation of the LAR values divided by their mean. Let $F$ be the set of all local assignment ratio values of users system fairness, $\mathcal{F}$, is formalized as:

$$
\mathcal{F}=\frac{\sigma(F)}{\mu(F)}
$$

Candidacy Constraint: Recall $w_{i} \cdot T_{a c c}$ is the set of tasks worker $w_{i}$ has accepted. A given task $t_{j}$ can be assigned to worker $w_{i}$ only if the system nominated her for the task, and she accepted the offer: $t_{i} \in w_{i} \cdot T_{a c c}$.

Capacity Constraint: Definition of the capacity constraint is intuitive. The number of tasks assigned to a worker cannot exceed her capacity: $\left|w_{i} \cdot T_{\text {all }}\right| \leq w_{i} . c$.

Assignment Ratio Constraint: Note that each task is offered
TABLE 1: Commonly Used Notation

\begin{tabular}{|l|l|}
\hline Notation & Explanation \\
\hline $\mathrm{h}[b, e]$ & time period; beginning from $b$, ending at $e$ \\
\hline$w\{A, c, f\}$ & $\begin{array}{l}\text { Worker; consists of set of availabilities } \\
(w . A), \text { capacity }(w . c) \text { and local assignment } \\
\text { ratio }(w \cdot f)\end{array}$ \\
\hline$t\left\{h_{s}, l_{s}, h_{r}, l_{r}, m\right\}$ & $\begin{array}{l}\text { task; consists of source location }\left(t . l_{s}\right) \text {, source } \\
\text { time period }\left(t . h_{s}\right), \text { destination location } \\
\left(t . l_{r}\right), \text { destination time period }\left(t . h_{r}\right) \text { and a } \\
\text { reward }(t . m) .\end{array}$ \\
\hline$a_{i p}\{h, l, r\}$ & $\begin{array}{l}p^{t h} \text { availability of } i^{t h} \text { worker } w_{i} \text { consists of } \\
\text { a time period } h, \text { a location } l \text { and radius } r\end{array}$ \\
\hline $\mathcal{T}, \mathcal{W}, \mathcal{A}, F$ & $\begin{array}{l}\text { set of all tasks, workers, availabilities and } \\
\text { local assignment ratios respectively. }\end{array}$ \\
\hline$T_{a l l}, w_{i} \cdot T_{a l l}$, & set of allocated tasks, allocated tasks to \\
$w_{i} . T_{a c c}$ & worker $w_{i}$ and tasks accepted by $w_{i}$. \\
\hline
\end{tabular}

to a set of nominees, yet among the candidates who accept the offer, only one worker will be selected. To forestall spamming of workers and avoid unnecessary communication costs, we avoid broadcasting offers to nominees. At the one extreme, the task can be repeatedly unicasted until one nominee accepts it. However, this approach would cause potentially long wait times. Therefore, we present a hybrid solution: multicasting the offer to $k$ workers, which avoids spamming of the workers while increasing the probability of at least one nominee accepting the offer. The value of $k$ is calculated for each task independently. In the next section, we show that higher values of $k$ will result in higher number of candidates, but it leads to a large set of spammed workers. To be able to define an upper limit, we introduce a systemwide metric called assignment ratio $(A R)$, which is the ratio of the number of allocated tasks over the number of accepted offers. Higher $A R$ indicates more accurate nominee selection, or less number of spammed workers. Therefore, we constraint assignment ratio to be higher than a predefined threshold $\theta$. We formalize the $A R$ as follows:

$$
A R=\frac{\left|T_{a l l}\right|}{\sum_{i=1}^{|\mathcal{W}|}\left|w_{i} \cdot T_{a c c}\right|}
$$

And define assignment ratio constraint as $A R \geq \theta$. Later, we will discuss relaxing this constraint to decrease the wait time of the customers.

\section{Allocation Model}

In this section, we describe the details of the 2-phase allocation model. Inputs to the system are the tasks from the customers, and the availabilities from the workers, both with time and location components. In many practical cases, both the tasks and the availabilities are registered in advance. Hence, one needs to check if a worker is still willing to do the job. Our model is able to do this check to increase the reliability of the worker with respect to the completion of the given task. In the next section, we also cover the online allocation scenario, in which tasks and availabilities can appear anywhere, anytime.

In the remaining parts of this section, we give the details of the 2-phase model in three steps: $(i)$ nomination of workers, (ii) batched progressive offer strategy, and (iii) task allocation. 

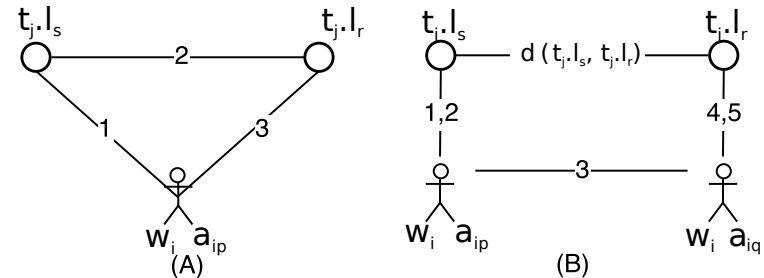

Fig. 2: Movement cost calculation of a task is different depending on the number of availabilities satisfying it. Roads are numbered with respect to travel order.

\subsection{Nomination of Workers}

Initial step of the 2-phase allocation procedure is to find out the appropriate set of workers to offer the task. These workers are called nominees. For a worker $w_{i}$ to be nominated to a task $t_{j}$, her availability set, $w_{i}$.A, should contain the necessary availabilities to satisfy both steps of $t_{j}$. Recall since tasks are in the form of spatio-temporal deliveries, one can divide them into two: receive and deliver steps. Satisfying one of these parts, lets assume receive, means that worker $w_{i}$ should have at least one availability $a_{i p}$, such that time period of task $t_{j} . h_{s}$ intersects with time period of availability $a_{i p} . h$ and source location $t_{j} . l_{s}$ lies inside the region $C r\left(a_{i p} . l, a_{i p} . r\right)$.

One can formalize the satisfaction relation between a step, receive or deliver, of a task $t_{j}$ and an availability $a_{i p}$ of a worker as a function $S$ :

$$
\begin{array}{r}
S\left(t_{j} . h_{s}, t_{j} . l_{s}, a_{i p}\right) \equiv\left(t_{j} . h_{s} \cap a_{i p} . h \neq \varnothing\right) \wedge \\
\left(t_{j} . l_{s} \in C r\left(a_{i p} . l, a_{i p} . r\right)\right)
\end{array}
$$

The intuition behind dividing the tasks into two steps is that, a worker can have separate availabilities such that one satisfies the requirements of the receive step and the other satisfies the deliver step. Given the set of workers, the system searches for those workers that have satisfying availabilities for both steps of a task. To formulate this relation, we employ the $S$ function to locate the pair of availabilities of a worker $w_{i}$ that satisfy the receive and deliver steps, respectively. We denote the resulting function as $N\left(t_{j}, w_{i}\right)$. If no such pair can be located, then the function produces an empty set.

$$
N\left(t_{j}, w_{i}\right)= \begin{cases}\left\{a_{i p}, a_{i q}\right\} & \exists a_{i p} \in w_{i} \cdot A \mid S\left(t_{j} . h_{s}, t_{j} . l_{s}, a_{i p}\right) \wedge \\ \varnothing & \exists a_{i q} \in w_{i} \cdot A \mid S\left(t_{j} . h_{r}, t_{j} . l_{r}, a_{i q}\right) \\ & \text { otherwise }\end{cases}
$$

We should note that, in Equation $7, p$ and $q$ values can be equal, which means a single availability might satisfy both requirements.

It would be unrealistic to assume that all nominated workers will have the same probability to accept the offered task. Besides availability, there are many factors that influence such a decision. Existing research showed that workers are willing to accept the tasks that are less costly for them and closer to their home locations [2]. Therefore, the acceptance probability of each nominated worker for a given task is negatively correlated with the task's cost, which is the movement cost in our case.
Many existing local delivery systems use the distance between the source and the destination as an indicator of the payment amount. It means that, tasks which require longer traveling pay more to their workers. On the other hand, the movement cost of a task might differ between workers, as they should move towards the source from their current location or move back to their previous location from the destination. Since we do not track the workers' locations, we assume that a worker is at the location provided as part of her valid availability and she tends to go back to that location, after completing the deliver step. We also assume that the acceptance does not depend on the previous acceptances or rejections.

Figure 2 shows two different scenarios regarding the calculation of the movement cost of a task. Let us define the movement cost of a task as $\alpha+\beta$, and let $d$ be the function that calculates the distance between two geo-spatial points. The distance between source and the destination, given by $\alpha=d\left(t_{j} . l_{s}, t_{j} . l_{r}\right)$, remains the same no matter which worker is assigned to the task. On the other hand, the distance traveled towards the source and from the destination, $\beta$, depends on the worker availabilities. In the first scenario, single availability of the worker, $a_{i p}$, satisfies both of the steps of the task. Therefore, the worker will only move towards the source, and from the destination $\left(\beta=d\left(a_{i p} . l, t_{j} . l_{s}\right)+d\left(t_{j} . l_{r}, a_{i p} . l\right)\right)$. In the second scenario however, after moving towards the source, as well as after delivering the item, worker will go back her initial position. Moreover, distances between the locations of those availabilities should be considered as well. Consequently, workers movement in the second scenario would be equal to $\beta=2 \times d\left(a_{i p} . l, t_{j} . l_{s}\right)+2 \times d\left(t_{j} . l_{r}, a_{i q} . l\right)+d\left(a_{i p} . l, a_{i q} . l\right)$.

The reward of the task is positively correlated with $\alpha$ and indifferent to who completes the task. Minimum value for the total travel distance is $2 \times \alpha$, which happens when the worker is already at the source location, or at the destination location, or lies on the linear line connecting these two points, i.e. $\alpha=\beta$. Intuitively, workers are willing to accept tasks with high income and less movement. Therefore, acceptance probability of a task $t_{j}$ by worker $w_{i}$, denoted as $R\left(t_{j}, w_{i}\right)$, is positively correlated with $\alpha$, but negatively correlated with $\beta$. For the ideal case, $\alpha=\beta$ acceptance probability should be 1 . We formalize this model in Equation 8. To take into account the probability of a worker refusing an offer even when she is a perfect match, we use a constant $c$, where $0 \leq c \leq 1$.

$$
R\left(t_{j}, w_{i}\right)=e^{\alpha-\beta} \times c
$$

Since $\alpha \leq \beta$ and $0 \leq c \leq 1$ the value of $R$ is guaranteed to lay between 0 and 1 . Selection of nominees for a task $t_{j}$ outputs the set $L\left(t_{j}, \mathcal{W}\right)$, which contains the worker $w_{i}$ and acceptance probability $R\left(t_{j}, w_{i}\right)$ pair. This set is used in our batched progressive offer strategy, a technique we implement to minimize the waiting time, while maximizing the assignment ratio.

$$
L\left(t_{j}, \mathcal{W}\right)=\left\{w_{i}, R\left(t_{j}, w_{i}\right) \mid w_{i} \in \mathcal{W} \wedge N\left(t_{j}, w_{i}\right) \neq \varnothing\right\}
$$

Let $\mathcal{A}$ be the set of all availabilities. Brute force approach to construct $L\left(t_{j}, \mathcal{W}\right)$ for a given task $t_{j} \in \mathcal{T}$ iterates 
through the availabilities set $\mathcal{A}$ and calculates $R\left(t_{j}, w_{i}\right)$ if $N\left(t_{j}, w_{i}\right) \neq \varnothing$, for each worker $w_{i} \in \mathcal{W}$. For all tasks, complexity of this calculation is $\mathcal{O}(|\mathcal{T} \| \mathcal{A}|)$. To decrease the computation time, we index availabilities on the temporal dimension using interval trees. For each task, we query this index two times, one for the receive step, and one for the deliver step. We check the spatial intersection only for the resulting availabilities. In the Online Task Allocation section, we discuss how long to wait between task arrival and nominee selection processes.

\subsection{Batched Progressive Offer Strategy}

There are three different approaches regarding offering a task to a predefined set of nominees. The first approach is broadcasting the offer. To forestall spamming of workers and avoid unnecessary communication costs, we avoid broadcasting offers to nominees. Moreover, we want to minimize the number of cases where a worker accepts the incoming offer but is not allocated to the task, as this might cause churn over time. Therefore, the ideal case is when there is exactly one candidate. Reaching this ideal case is only possible with unicasting the offer. However, there is the probability of the nominee rejecting the offer, even though she is available. Therefore, the system should follow a progressive approach, by offering the task one by one, until somebody accepts, and waiting for a preset time between each round. Obviously, this will result in a long waiting time for the customer to see if his task is going to be served or not. To avoid both situations, we multicast the offer to $k$ nominees in batches, progressively, until there is at least one candidate. With this approach, we decrease the waiting time of the customer, while limiting the number of candidates. For each task, once the value of $k$ is calculated, it is used for further batching, if necessary.

Let us call the probability of the task being accepted by at least one nominee as probability of response. For each task $t_{j}$, the probability of response, when offered to $k$ nominees, can be calculated using the probability of all $k$ nominees refusing it. Recall that $L\left(t_{j}, \mathcal{W}\right)$ is the set of nominee and acceptance probability pairs, and assume that it is sorted by the acceptance probability values. Probability of response for a batch of $k$ workers is calculated as follows.

$$
P\left(k, L\left(t_{j}, \mathcal{W}\right)\right)=1-\prod_{i=1}^{k}\left(1-\left(R\left(t_{j}, w_{i}\right)\right)\right)
$$

By keeping the value of $P\left(k, L\left(t_{j}, \mathcal{W}\right)\right)$ above a tuning parameter $\epsilon$, the lower bound of the $k$ can be defined. We call $\epsilon$, the threshold of probability of response.

The upper bound, however, is calculated using the assignment ratio as a constraint. To limit the value of $k$, first we should be able to predict how many nominees are likely to accept the offer. In the worst case for assignment ratio all $k$ nominees accept the offer. As we try to maximize the assignment ratio, the number of candidates should not exceed a certain number. Although it is not realistic to expect that all $k$ workers will accept the offer, it is still useful to limit the $k$ value.

Given a task $t_{j}$, let us define the probability of $i^{t h}$ worker accepting the offer as a random variable $x_{i}$. Then the expected value of it is $E\left[x_{i}\right]=R\left(t_{j}, w_{i}\right)$. Transforming into multiple workers, assignment ratio would be one over the number of candidates, therefore, it would be equal to $E\left[\frac{1}{x_{1}+x_{2}+\ldots+x_{k}}\right]$. From probability theory, it is known that $E\left[\frac{1}{x_{1}+x_{2}+\ldots+x_{k}}\right] \geq \frac{1}{E\left[x_{1}+x_{2}+\ldots+x_{k}\right]}$, thus the latter could be used as a lower bound for the expected value, where, $E\left[x_{1}+x_{2} \ldots+x_{k}\right]=R\left(t_{j}, w_{1}\right)+R\left(t_{j}, w_{2}\right)+\ldots R\left(t_{j}, w_{k}\right)$. With this at hand, let us define a function $\mathcal{E}\left(k, L\left(t_{j}\right)\right)$ as a lower bound for the expected value of the assignment ratio:

$$
\mathcal{E}\left(k, L\left(t_{j}, \mathcal{W}\right)\right)=\frac{1}{E\left[x_{1}+x_{2}+\ldots+x_{k}\right]}
$$

Recall one of the constraints is keeping the assignment ratio of the system above a predefined threshold $\theta$. We use the same threshold, as a lower bound to function $\mathcal{E}\left(k, L\left(t_{j}, \mathcal{W}\right)\right)$ to satisfy assignment ratio constraint locally, for each allocation. Although local satisfaction is stronger than the global constraint, as we will discuss next, the assignment ratio constraint is relaxed when it contradicts with the probability of response.

While the probability of response $P\left(k, L\left(t_{j}, \mathcal{W}\right)\right)$ is positively correlated with $k$, as it is shown above, the expected value of the assignment ratio (value of $\mathcal{E}\left(k, L\left(t_{j}, \mathcal{W}\right)\right)$ ) decreases with it. Therefore, bounding $\mathcal{E}$ function from below, sets an upper bound on the value of $k$. With the above defined thresholds, the value of $k$ should guarantee that probability of response $P\left(k, L\left(t_{j}, \mathcal{W}\right)\right)$ is above $\epsilon$, while the assignment ratio constraint is satisfied for each task, i.e. $\mathcal{E}\left(k, L\left(t_{j}, \mathcal{W}\right)\right)$ is above $\theta$.

In summary, $k$ is selected using following inequality:

$$
\begin{aligned}
& k \geq \min \left\{k \mid\left(P\left(k, L\left(t_{j}, \mathcal{W}\right)\right) \geq \epsilon\right\}\right. \\
& k \leq \max \left\{k \mid \mathcal{E}\left(k, L\left(t_{j}, \mathcal{W}\right)\right) \geq \theta\right\}
\end{aligned}
$$

$k$ is set to the maximum value that satisfies both of those inequalities. However, the given inequality might be invalid with respect to selection of the $\epsilon$ and $\theta$ values. Consider that, even the smallest $k$ value satisfying the upper inequality might not satisfy the lower one. In that case, we relax the assignment ratio constraint, and use the $k$ that satisfies the upper inequality.

After this calculation, the task is offered to the first $k$ workers and the system waits for a predefined time. Recall that, workers are sorted in the decreasing order of the acceptance probability. In case of all nominees refuse the offer, the task is offered to the next $k$ workers, until there is at least one candidate or all nominees are asked.

\subsection{Task Allocation}

The last step of the 2-phase model is to select one worker among the candidates to allocate the task.

Figure 3 shows an example scenario in which there are three tasks and three candidates. The edges are from tasks to their candidates. For example $t_{1}$ is accepted by $w_{1}$ and $w_{2}$, while $w_{1}$ is the only candidate for $t_{2}$. The simplest version of this problem is finding a one-to-one assignment scheme of tasks to workers, assuming all the edges have equal weight. By definition, this is the bipartite graph assignment problem. In our specific case, there is a one-to-many relationship between the tasks and the workers. Moreover, to capture the spatial aspect of the problem, one might want to use 


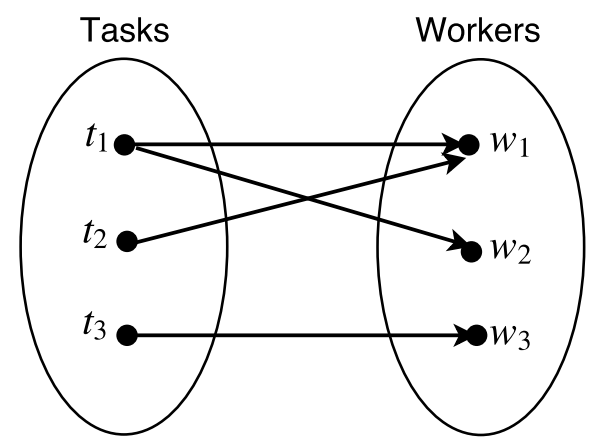

Fig. 3: Allocation of tasks to candidates

edge weights to represent movement cost and add capacity to workers as well. Including these additional constraints, the task-to-worker bipartite graph can be represented as a flow graph by adding a source and a sink. With this representation, task to worker allocation can be reduced to the minimum cost flow (MCF) problem and solved optimally with well known algorithms, i.e. successive shortest path or cycle canceling [21].

Even though they reach optimal result on task allocation, minimum cost flow based solutions have their own drawbacks. First of all, running time of the optimal solution is far from being feasible because of high complexity. Given the task to candidate bipartite graph $G(E, V)$, s.t. $V=\mathcal{T} \cup \mathcal{W}$ and $E$ represents the acceptances, the complexity of a careful implementation of successive shortest path algorithm is $\mathcal{O}(|V||E| \log |V|)$. Second, with the aforementioned definition of fairness, it is hard to integrate it into the MCF-based algorithms. This is because at each iteration $L A R$ values are updated, which updates the cost-matrix as well.

Assuming that $L A R$ values are static, introducing fairness as a new constraint transforms the problem to minimum cost maximum flow problem. The goal of this problem is to select the minimum cost flow among multiple maximum flows. In our setting, this corresponds to maximizing task allocation ratio while minimizing unfairness objectives. This problem could be solved with Hungarian algorithm, however, similar to the previous discussion, the high complexity of the algorithm, $\mathcal{O}\left(n^{3}\right)$, makes it inefficient for online allocation scenarios [24].

The naïve approach for allocation is random selection of one candidate, which is used as one of the baselines in our experimental evaluation. On the other hand, to capture the spatial aspect of crowdsourced delivery, it is beneficial to allocate the nearest worker [21]. This approach can be extended by proactive allocation of workers, if distribution of tasks is known in advance [25]. Last but not the least, inspired from file allocation techniques from the operating systems literature, selecting the Least Allocated Worker first could increase the Task Allocation Ratio by reserving room for further allocations. While all approaches are feasible in terms of their running time, our experimental evaluation shows that they either fell short in terms of modeling the fairness or have subpar performance with respect to task allocation when the capacity is constrained.

To cope with the aforementioned challenges, we introduce an algorithm called F-Aware, given in Algorithm 1. It is a greedy algorithm that allocates tasks to workers one by

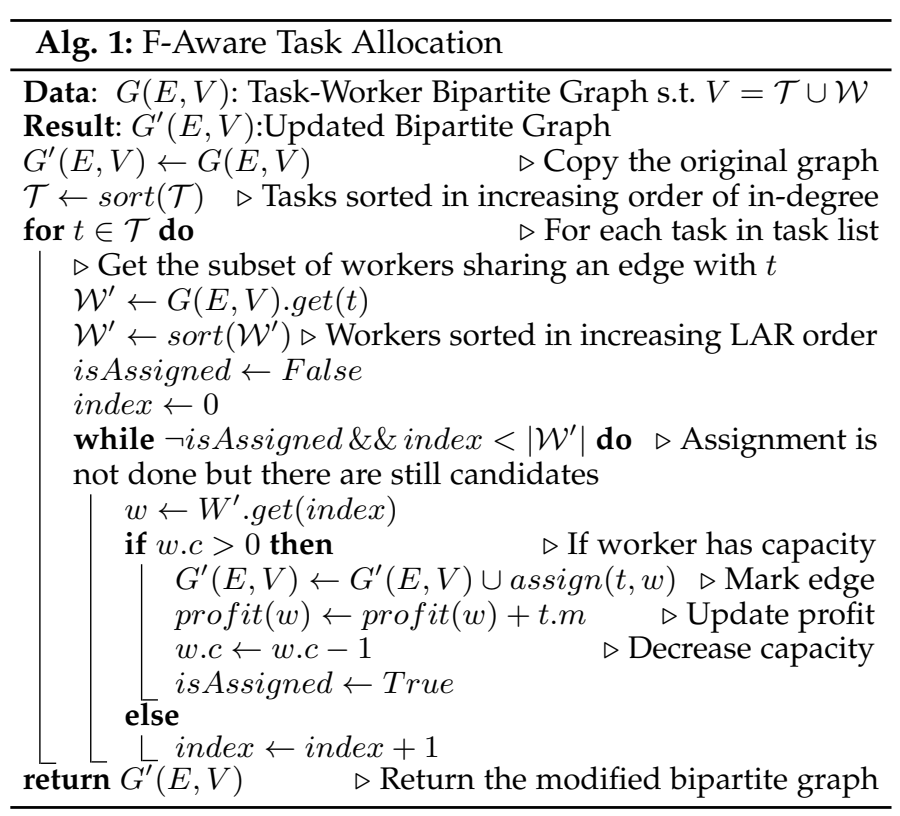

one. Given the task-to-worker bipartite graph $G(E, V)$, s.t. $V=\mathcal{T} \cup \mathcal{W}$, it considers tasks in the increasing order of the node in-degree. Tasks with less candidates are placed first, as the ones with higher degree have more flexibility. For each task, each worker with remaining capacity higher than 0 is considered as a candidate and the one with the lowest local assignment ratio is selected. If there are more than one worker with the same $L A R$, the one with the higher denominator is selected. Recall that if the capacity of a worker, $w_{i} . c$, is lower than the number of tasks she has accepted, we only consider the first $w_{i} . c$ offers. The process continues until all tasks are visited. When a task $t_{j}$ is assigned to a worker $w_{i}$, the input graph, the total earnings, and the residual capacity of $w_{i}$ are updated accordingly.

Our experimental results confirm the effectiveness of $F$ Aware in terms of running time, task allocation ratio, and fairness it achieves.

\section{OnLine Task Allocation}

While in offline allocation based applications, all the tasks and availabilities are known in advance, in real-time environments, they can appear at anytime, anywhere [36]. Consider an example scenario, in which a customer would like to travel to airport and asks the crowd for a ride. Overnight reply to this request would be too late, as the user would take a taxi after waiting for a relatively small amount of time. In fact, these dynamically arriving requests require online processing. Therefore, the problem of allocating tasks to workers in a dynamically changing environment raises.

While aforementioned example requires instant response to a customer, there are also some crowdsourced delivery applications that allow decision maker system to wait for a period, before allocating the task. Online shopping is an example of such applications. In this case, a seller can wait to group deliveries by their destinations and allocate only one worker for multiple packages. During this waiting period, system collects mini-batches of tasks and worker availabilities for a period and they are processed against 
each other when the period expires. In this section, we present our modifications on the 2-phase allocation model to cover both instant and mini-batch allocation scenarios.

Figure 1 shows the illustration of the workflow for the 2-phase allocation model. Our first modification for adapting this workflow to online task allocation is adding two windows on the selection of nominees. These windows are for the tasks and for the availabilities, respectively. They are neither sliding nor tumbling windows. When a task or availability arrives, it is appended into the corresponding window. An availability is removed from the window when it expires. A task on the other hand can be removed under two conditions: $i$ ) it is assigned to a worker, ii) it expires. However, waiting until expiration of a task before allocation might cause misses, as all satisfying availabilities might expire meanwhile. Therefore, we also define a window size, in terms of minutes. For every window expiration, the tasks are processed against availabilities and nominees are identified. After this step, batched progressive offer and task allocation steps are used as they are. There are two corner cases about tasks. First, if a task has no nominees at the time of the window expiration, it stays in the window and participates in the following window expirations, until its own expiration. Second, if the task has nominees but all of them rejected the offer, it again remains in the window for new nominees to arrive. The same task is never offered to the same nominee more than once.

There are multiple constraints on the window size decision. First of all, it should be shorter than the smallest time period of the set of all tasks and availabilities to guarantee processing. Second, the waiting time should not exceed reasonable response time of the application. An online shopping application that needs 2-hours delivery guarantee cannot define the window size as 3 hours. For applications that require instant reply, the window size can be set to 0 . In instant task allocation, when a task arrives, it is processed against the availabilities window to identify nominees. If there are not any, it is added to the tasks window and stays there until its expiration time. When an availability arrives, all tasks and availabilities present in the window are processed, since this availability might be completing a partial match. A partial match is possible when a worker has a satisfying availability for only one of the steps of the task. We leave the detailed discussion of partial match processing as future work. In our experimental evaluation, we study the feasibility and effectiveness of our allocation model with various window sizes, including instant allocation, i.e. window size equals to 0.

\section{Experimental Evaluation}

In this section, we present the detailed evaluation of our proposed 2-phase allocation model and the fairness-aware task allocation algorithm, F-Aware.

To easily determine the superiority of a solution over other solutions, we combine optimization goals into a single parametric objective function $\mathcal{O}$ and define it as:

$$
\mathcal{O}=T A R \times e^{-(\rho * \mathcal{F})}
$$

Since the goals are maximizing the TAR while minimizing the unfairness, the objective function is proportional to
TAR. But the exponential part of it is inversely proportional with the global unfairness metric $\mathcal{F}$. To enable the system to prioritize one component of the objective over the other, we introduce the parameter $\rho$, where $0 \leq \rho \leq 1$. By setting $\rho$ to 0 , one can simplify this objective to task allocation ratio only. Higher values of it will increase the importance of the unfairness in the overall objective.

The evaluation includes four sets of experiments. In the first set, we compare F-Aware with 4 other competitor algorithms in terms of task allocation ratio (TAR), unfairness and value of the objective function. Recall that the objective function, as defined in Eq. 13, is the combined metric of TAR and unfairness. The naïve approach of assigning tasks to workers is random selection among the candidates, referred to as Random in the performance graphs. The second approach is to select Least Allocated Worker First (LAF). The intuition behind this approach is trying to reserve room for further task allocations. Existing work of task allocation in spatial crowdsourcing mostly use Nearest Neighbor Priority strategy [21], [25] to capture the spatial-aspect of the problem. [21] introduces allocation techniques based on location entropy, and [25] extends nearest worker priority technique with pro-active deployment of workers to geo-grids. We only prioritize the nearest worker since we do not make assumptions on distribution of tasks. This algorithm is referred to as Nearest in this section. Lastly, we use successive shortest paths algorithm which solves the minimum cost flow(MCF) problem [23]. This algorithm is optimal on Task Allocation Ratio, hence it is used to evaluate TAR of all algorithms. The second set of experiments studies the efficiency of our batch incremental offer strategy, that is how different values of $\epsilon$ and $\theta$ affect the $k$ value, thus assignment ratio, and unfairness. The third set studies online task allocation, presenting task allocation ratio and unfairness as a function of window size. Finally, the last set is the sensitivity study that presents task allocation ratio and unfairness of different time period lengths as a function of coefficient of mean. As we will detail soon, length of the time period and coefficient of the mean are two variables we use to adapt real-world data to our setup.

We implemented all algorithms using Java 1.8. All experiments were executed on a Linux server with 2 Intel Xeon E5520 2.27GHz CPUs and 64GB of RAM.

Dataset. Experiments are performed using two realworld datasets. The first dataset contains the Foursquare check-ins from New York City for the month of May $2012[34]^{6}$ This dataset contains around 50,000 check-ins from 987 different users. The second dataset is a taxi-trip dataset for Manhattan, for the same time period 7 We used up to 512,000 randomly sampled rows from the taxi dataset. Half million tasks for a city, for one month, is a fair workload, considering assignment could be done independently for each city. Each row of the check-in data contains a user id, time of the check-in, and the location of it. Each row in the taxi dataset contains the time and the location of the pick-up and the drop-off. It also contains the cost of the trip. To simulate crowdsourced delivery behavior, we use taxi trips as tasks and check-ins as spatio-temporal availabilities

6. sites.google.com/site/yangdingqi/home/foursquare-dataset 7. www.nyc.gov $/ \mathrm{html} / \mathrm{tlc} / \mathrm{html} /$ about/trip_record_data.shtml 


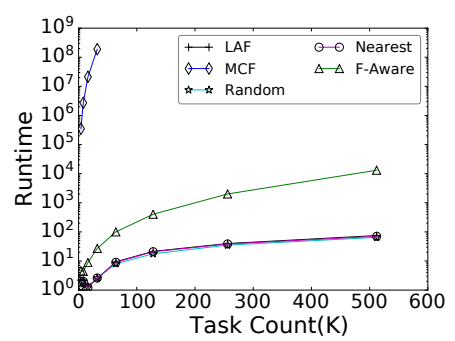

(a) Runtime

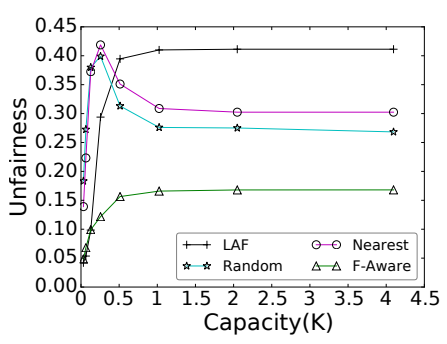

(a) Unfairness

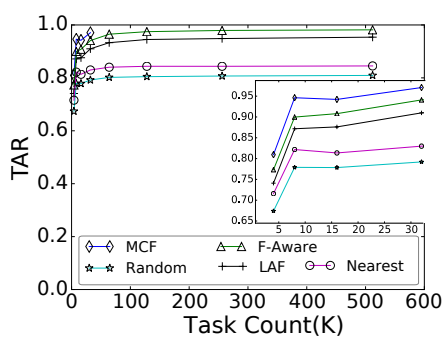

(b) TAR

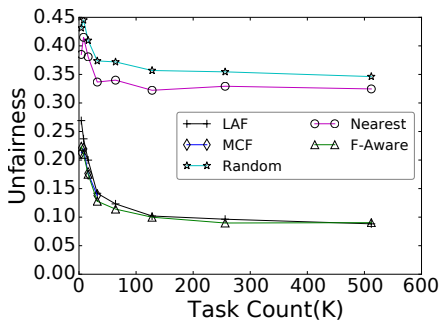

(c) Unfairness

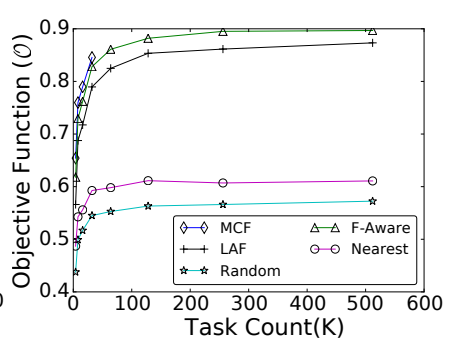

(d) Objective

Fig. 4: Scalability

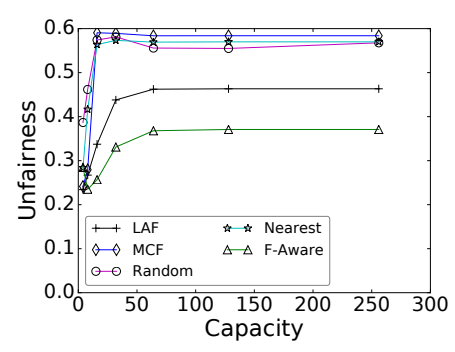

(b) Unfairness-small task set

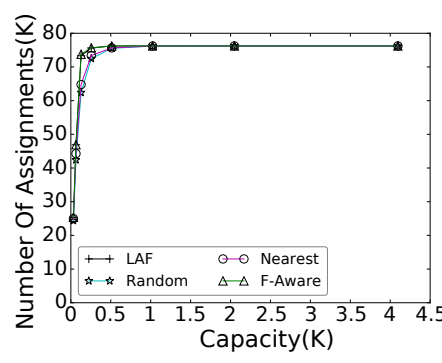

(c) \# Of Completed Tasks

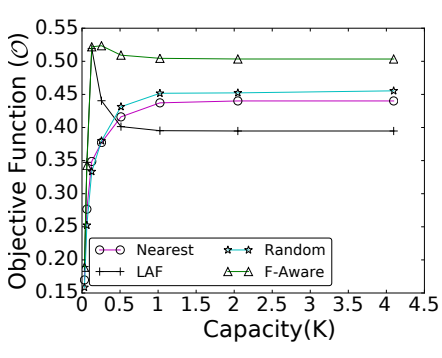

(d) Objective

Fig. 5: Effect of the Worker Capacity

of the workers. For both datasets, we converted all time points to time ranges by adding time periods. Given a point in time, $p$, a period of length $\Delta T$ is created with beginning and end points $[p, p+(\Delta T)]$. We treat pick-up location and time of a taxi trip as the task's source location and source validity time. The same applies to drop-off location and time and tasks deliver location and validity. For each check-in, we use the location of it as the location of the availability and converted the time of it to a time period as described above. Radius of each availability is sampled from a Normal distribution with mean and standard deviation calculated using taxi trips. We study the effect of this conversion as part of our last experiment presented in this section, by varying the mean size of the time period. For all other experiments, the mean value for the time period is taken as 2 hours. Last, we used the cost of the trip as the reward of the task.

\subsection{Scalability}

In this set, we present two subsets of experiments. In the first subset, we observe the running time performance, task allocation ratio, and unfairness as a function of the number of tasks. The number of tasks is doubled for every data point ranging from 4000 tasks up to 512,000 . Capacities of the workers are assigned using a Normal distribution and the mean is set to the number of tasks over the number of workers. Standard deviation of the distribution is set to mean over 4 to make sure all capacities are at least 0 . Using this tight capacity assignment for this particular experiment set, we ensure that the capacity is barely enough for allocating all the tasks. This gives a clear advantage to algorithms that can allocate tasks close to optimal. In the second subset, we observe the task allocation ratio and unfairness as a function of worker capacity. 128,000 tasks are used, and capacities are doubled for every data point ranging from 32 to 4096 . To observe the behavior of the $M C F$ algorithm, we also present unfairness as a function of capacity using 8000 tasks (Figure 5b). In this experiment, capacities are ranging from 4 to 256 .

Figures 4 and 5 present our scalability related results. In all figures, the $x$-axis represents either the number of tasks to allocate, or the capacity of workers, and $y$-axis represents the performance metric. Different series represent different assignment algorithms. Figure $4 a$ plots the running time as a function of the number of the tasks. We make two observations from the figure. First, and most importantly, $F$-Aware runs $10^{7}$ times faster than the Minimum Cost Flow $M C F$ algorithm. For 32,000 tasks, the running time of the MCF is $1.89 \times 10^{8}$ milliseconds while F-Aware completes in 27 milliseconds. Because of the long evaluation time, we do not present $M C F$ results for more than 32,000 tasks. Secondly, the running time of the $F$-Aware algorithm is linear with the task count. Increasing from 4000 tasks to 512,000 tasks, the running time increases from 4.6 milliseconds to 13,130 milliseconds. The difference between the running times of Random, LAF and Nearest assignment algorithms are negligible.

Figure $4 \mathrm{~b}$ plots the task allocation ratio as a function of the number of tasks. To increase readability, it also includes the zoomed small figure of data points between $[4000,32,000]$. Since any allocation algorithm will left tasks with no candidates unassigned, in this experiment we consider only tasks with at least one candidate. We observe that all allocation algorithms are able to hold their allocation ratio with the increasing number of tasks. The most important observation is that F-Aware is able to assign $96.9 \%$ of the tasks that are allocated by MCF. For 32,000 tasks, MCF reaches to $97 \%$ task allocation ration, while F-Aware allocates $94 \%$ of all tasks. When we double the number of tasks, F-Aware still allocates $96.5 \%$ of tasks, while Random and Nearest worker allocation algorithms stay at $80 \%$ and $84 \%$, respectively. In terms of TAR, $L A F$ is the best competitor of F-Aware. This is expected as its goal is to increase 
the number of allocated tasks. However, TAR of F-Aware is still higher. For 512,000 tasks, F-Aware reaches to $98.1 \%$ task allocation ratio, while $L A F$ reaches to $95.3 \%$.

We discuss the Figures $4 \mathrm{c}, 5 \mathrm{a}, 5 \mathrm{~b}$ and $5 \mathrm{c}$ together as they are complementary. First three figures plot the unfairness as a function of task count, capacity and capacity respectively. The last one represents the number of assigned tasks, as a function of capacity. In Figure 5b we use only 8000 tasks to include $M C F$ algorithm. In the first Figure, $4 \mathrm{c}$, we use up to 512,000 tasks but exclude MCF after 32,000 tasks, because of the impractically long running time. For the remaining two, we use 128,000 tasks, and capacity of the workers varies from 32 to 4096 .

At this point, it would be useful to recall, $L A R$ of a worker is the ratio of revenue she made from completed task over total reward of the offers she has accepted. However, since capacity limits the assignable task count, if a worker $w_{i}$ accepts more offer than her capacity $w_{i}^{c}$, we only consider first $w_{i}^{c}$ offers when calculating LAR. Therefore, when evaluating performance of an algorithm in terms of fairness, one must consider two cases when capacity limits assignments, and when there are more than enough room for assignments.

When worker capacities are enough to serve all tasks, we observe a significant difference between unfairness values. In Figure 5b, for small dataset, Random, Nearest, and MCF perform similar. In contrast, unfairness metric of $L A F 0.8 \times$ of the same metric of those three. We observe F-Aware, performs best among all. Unfairness metric $M C F$ algorithm is $1.5 \times$ that of our F-Aware algorithm when capacity is 256. For the larger task set, the difference between $L A F$ and $F$-Aware becomes even more significant (figures $5 \mathrm{a}, 5 \mathrm{c}$. When the capacity is set to 1024 unfairness value of $L A F$ is 0.41 while $F$-Aware has only 0.16 unfairness. For same data point Nearest, and Random have 0.30, 0.27 unfairness values respectively. We make two additional observations from this figure. First, up to 256 capacity, unfairness values increase. This is because capacity of workers less than number of acceptances. In this set of experiments each worker accepts 215 offers in average. After this point number of accepted tasks used when calculating $L A R$. Using Figure $5 \mathrm{c}$ we observe all algorithms reach maximum number of allocated tasks at capacity 1024, which reflected as stabilized unfairness values in Figure 5a Second, LAF performs poorer than Nearest and Random assignment approaches. Since it does not take user input into account, (i.e. accepting offers) when tasks are distributed evenly, workers who have accepted small number of offers have $L A R=1$, while workers with large number of acceptances have too low $L A R$ values.

When capacity is set to a too low value, we cannot observe significant difference between unfairness values of $M C F, L A F$, and F-Aware. This is because all workers are fully allocated. On the other hand, in Figure $4 \mathrm{c}$ values of unfairness metric for Random, and Nearest is around 3.6× that of F-Aware. The reason behind is system could serve less number of tasks (Figure $4 \mathrm{~b}$, when one of these two assignment algorithm is used.

Figures $4 \mathrm{~d}$ and $5 \mathrm{~d}$ present the performance in terms of our objective, Eq13. as a function of task count and capacity respectively. In these experiments, the $\rho$ parameter is set to 1 , to observe the balanced outcome of task allocation ratio and fairness. In Figure $4 \mathrm{~d}$, we observe that the difference between the objective values of F-Aware and MCF are negligible. FAware performs as good as $M C F$, in terms of our objective, with $10^{7}$ times faster processing speed. Another observation from the same figure is that, $F$-Aware performs $4 \%$ better than $L A F$, even with limited capacity. For 128,000 tasks, FAware has 0.86 objective value while $L A F$ has 0.82 . When there are more than enough room for assignments, we observe that the gap between F-Aware and LAF becomes even more significant, as shown by Figure $5 \mathrm{~d}$ For a capacity value of 4096 the objective value of F-Aware is 0.5 , while $L A F$ could reach only 0.39 . Objective values of Nearest and Random are better than $L A F$, because of the lower unfairness values, but still they are far from performing as good as F-Aware.

To summarize this experiment set, one can say that using $M C F$ is impractical due to its long running time. In contrast, F-Aware runs $10^{7}$ times faster. When only TAR is considered, LAF performs similar to F-Aware, but the other two approaches, Random and Nearest, leave $19 \%$ and $15 \%$ of all tasks unassigned, respectively. While $L A F$ is the best competitor of F-Aware, in terms of TAR and runtime, its unfairness metric value is $2.5 \times$ that of $F$-Aware and its objective value is $80 \%$ that of our F-Aware algorithm.

\subsection{Effect of the Batch Size}

We observe average $k$, unfairness, assignment ratio, and average wait time as a function of the probability of response threshold $\epsilon$. Figure 6 presents our batch size related results. In all figures $x$-axis represents the value of $\epsilon$ and $y$-axis represents a performance metric. Different series represent different assignment ratio thresholds, $\theta$. For all series we use the F-Aware assignment algorithm. For this set of experiments capacity of workers is high enough to prevent a bottleneck.

Figure 6a plots the change in the value of $k$ as a function of $\epsilon$. The series with circle marker is the unicast line, i.e. $k$ is set to 1 . Recall that the value of $k$ is bounded from below by a function of $\epsilon$ and bounded from above by a function of $\theta$. We select the largest $k$ inside this range. Higher values of $\epsilon$ and $\theta$ imply tighter bounds. Since $\theta=0.0$ means unlimited upper bound, practically it is the broadcasting line. We observe that as long as $\epsilon \leq 0.95$ the value of $k$ is limited by the assignment ratio threshold $\theta$. Increasing $\theta$ from 0.2 to 0.4 decreases the average $k$ value from 14 to 6 . Since task completion is our primary goal, when lower bound is higher than the upper bound (possible in some cases based on the definition of Equation 12), we use the lower bound for deciding the $k$ value. One can observe this behavior when $\epsilon \geq 0.95$, as all the average $k$ values are closer to broadcasting.

Figure $6 \mathrm{~b}$ plots the unfairness as a function of $\epsilon$. We observe that smaller $k$ values provides a more fair systems. The unfairness of unicasting is 0 , as whenever a worker accepts a task, she will be assigned to it. Whereas the unfairness of broadcasting is 0.36 . Most importantly, unfairness of multicasting the offer to an average of 5.8 nominees is 0.20 . There are two reasons behind this observation. First, local assignment ratio of a worker is negatively correlated with the number of her acceptances. Therefore, the mean of 


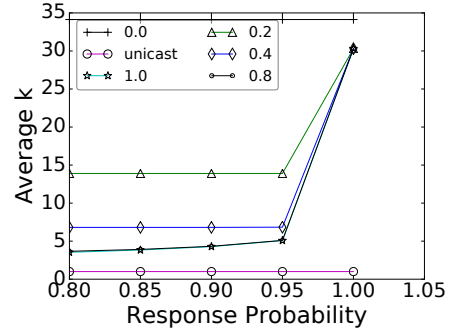

(a) Average K

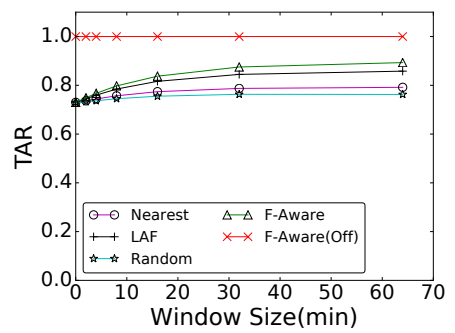

(a) TAR Rel. to Offline

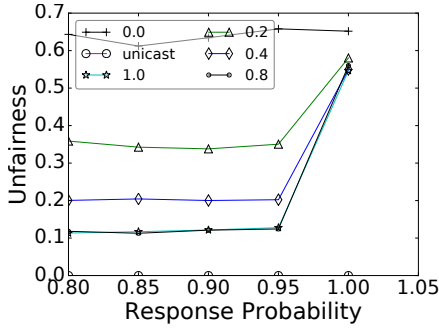

(b) Unfairness

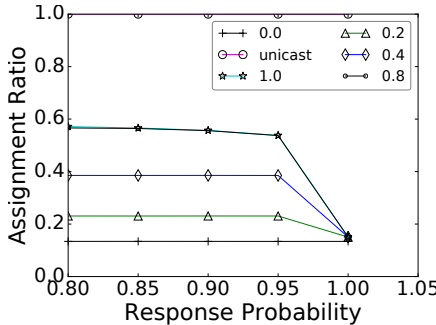

(c) Assignment Ratio

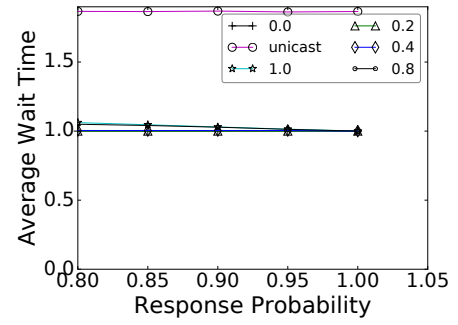

(d) Average Wait Time

Fig. 6: Effect of the Batch Size

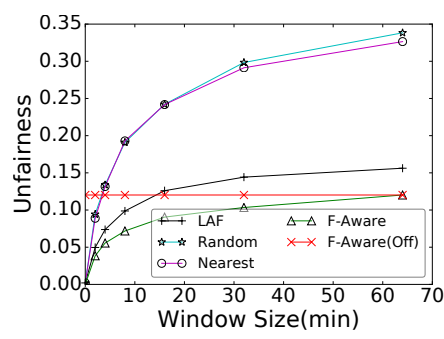

(b) Unfairness Rel. to Offline

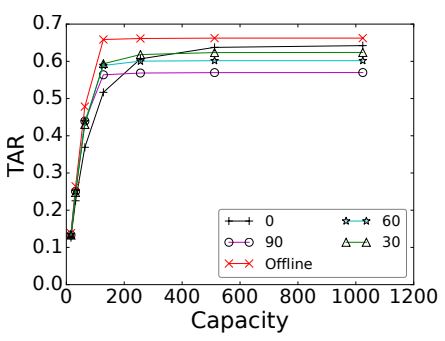

(c) TAR

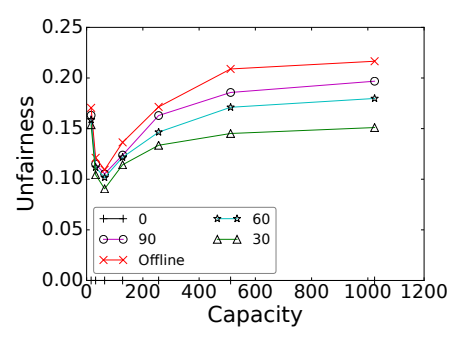

(d) Unfairness

Fig. 7: Online Task Allocation

the set of $L A R$ values increases, which leads to a decrease in the coefficient of variation metric. Second, and more importantly, when a worker accepts an offer, probability of her getting the job is higher with the smaller values of $k$. In the extreme case, that is unicasting, acceptance implies assignment, hence unfairness $=0$. We can also observe a similar behavior in Figure 6c Assignment ratio of the unicasting equals to 1 . For the other series, we can see that assignment ratio is negatively correlated with the average $k$ value. For broadcasting, it decreases up to 0.13 . When the average $k$ is 5.8 , the assignment ratio is 0.38 . Another important observation is theoretically $\theta=1.0$ should be the unicast line but it has 0.6 assignment ratio. The reason behind is $\epsilon$ is a stronger constraint than $\theta$. We do not observe same behavior lines other than $\theta=1.0$, and $\theta=0.8$

Figure $6 \mathrm{~d}$ plots the average wait time as a function of $\epsilon . y$-axis shows the average number of rounds passed until there is at least one candidate. Recall that the task is offered to nominees in batches until there is at least one response. Between each round, the system waits for a predefined period to let nominees decide. For some scenarios, e.g. on-demand transportation, customers expect almost instant reply. Higher number of rounds before acceptance leads to late notification to a customer. In case of broadcasting, since all nominees are notified at once, number of rounds for response is always 1 . However, as we have just seen, broadcasting leads to low assignment ratio and high unfairness. At the other extreme, that is unicasting, the average wait time is $1.8 \times$ of broadcasting. Multicasting is better than both approaches. Multicasting the task to an average of $5.08(\epsilon=0.8, \theta=0.95)$ nominees leads to only 1.01 average rounds, while providing only 0.13 unfairness.

In summary, one can say that multicasting is better than broadcasting in terms of assignment ratio and fairness. Moreover, it beats unicasting when average wait time is considered, while being almost as fair.

\subsection{Online Allocation}

We observe the task allocation ratio and unfairness as a function of the window size and as a function of capacity. Figure 7 shows our online allocation related results. For Figures $7 \mathrm{a}$ and $7 \mathrm{~b}$, the $x$-axis represents the window size in terms of minutes. In addition to window size equals to 0 , the $x$-axis also includes data points starting from 2 minutes, going up to 64 , doubling at each step. Different series represent different task allocation algorithms. For Figures $7 \mathrm{c}$ and $7 \mathrm{~d}$, the $x$-axis represents worker capacities. The values are doubled for each data point, starting from 16 , going up to 512 . For all figures, the $y$-axis represents a performance metric. In this set, we used 96,000 tasks.

Figure $7 \mathrm{a}$ shows the task allocation ratio as a function of the window size. The red line with cross markers represents offline F-Aware algorithm. All the other series are drawn relative to this line, therefore it is the $y=1$ line. We observe that for the instant task allocation, the difference between different series is negligible. This is because the number of nominees for each task is very small, and thus the decisions of the algorithms do not create any difference. On the other hand, when the window size is increased, we can see that $F$-Aware, and $L A F$ performs better than the other two approaches and gets closer to the offline assignment. For the 64 minutes window, F-Aware, and LAF allocates $88 \%$, and $85 \%$ of the tasks assignable by offline allocation, whereas Nearest, and Random allocation approaches stay at $79 \%$ and $76 \%$, respectively.

Figure $7 \mathrm{~b}$ plots unfairness as a function of the window size using the same setup as Figure $7 \mathrm{a}$. When smaller windows are used, since tasks are offered to only current nominees, the unfairness behavior is similar to using smaller $k$ values. For example, unfairness is 0 , when instant allocation strategy applied, as in unicasting. For longer window sizes, the results expected to become more similar to the offline setup. For example The unfairness of offline F-Aware is 


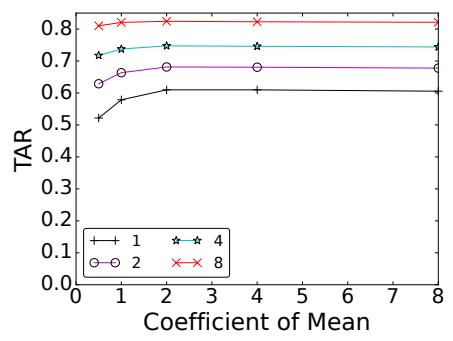

(a) Task Allocation Ratio

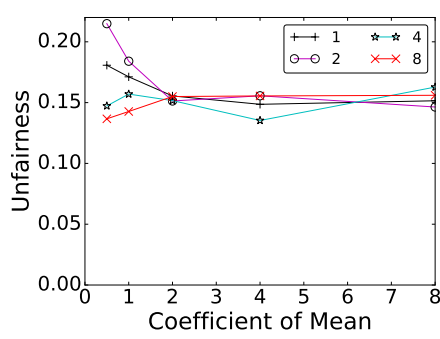

(b) Unfairness

Fig. 8: Sensitivity Experiment

0.13 , while online F-Aware increases from 0.04 to 0.11 when window size increased to 64 minutes from 2 minutes. The most important observation is F-Aware performs best among all online algorithms. For the window size of 64 minutes online F-Aware has 0.11 unfairness while $L A F$, Nearest, and Random have $0.15,0.32,0.33$ respectively. We also observe the other three algorithms are even less fair than offline $F$ Aware, for windows larger than 16 minutes.

Figures $7 \mathrm{c}$ and $7 \mathrm{~d}$ plot the task allocation ratio and unfairness as a function of worker capacity. Different series represent different window sizes, including offline and instant assignments. For all series, F-Aware algorithm is used. We make two main observations. First, the task allocation ratio for smaller window sizes is higher compared to larger window sizes. The reason behind this is that, some of the availabilities satisfying tasks expire before making a decision. At first sight, one might think larger window sizes should produce closer results to the offline scenario, but this is not the case. In the offline scenario all the information is known in advance, and decisions are made before expiration. In contrast, with large windows the availabilities might expire before processing. Second, larger windows result in less fair allocation. As we mentioned earlier, smaller windows behave like batches with smaller $k$ values. As batch-size experiments showed, when a task offered to less number of workers, fairness increases since sum of total accepted offers for each worker decreases.

\subsection{Sensitivity Experiments}

We observe the task allocation ratio and unfairness as a function of the coefficient of mean. Different series represent different $\Delta T$ values. Recall that these are the two parameters involved when we used the taxi trips as the tasks, and the check-ins as spatio-temporal availabilities of workers. The radius added to a check-in is sampled from a Normal distribution with mean and standard deviation set to that of the taxi trips. For each data point on the $x$-axis, the mean of this distribution is multiplied with the respective number. For all experiments, $y$-axis represents a performance metric.

Figure 8 a plots the task allocation ratio as a function of the coefficient of radius. We observe that $\Delta T$ has a great effect on the task allocation ratio. When coefficient of radius is 1 and $\Delta T=1,57 \%$ of the tasks are allocated, while this number is $82 \%$ when $\Delta T=8$ for the same coefficient. On the other hand, we cannot observe the same effect for larger radius values. Consider the $\Delta T=4$ line. The task allocation ratio increases by only $0.1 \%$ when the coefficient is increased from 1 to 4 .

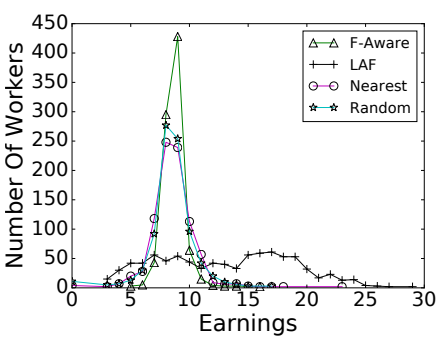

(a) Earning Distribution

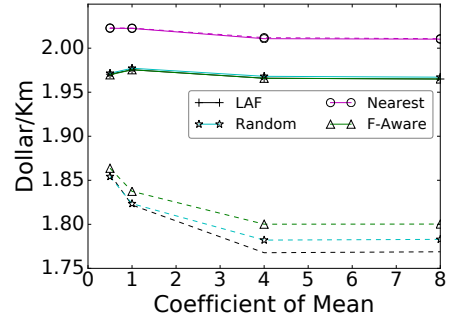

(b) Earnings of Workers Presented as Dollar per Kilometer

Fig. 9: Discussions
Figure $8 \mathrm{~b}$ plots unfairness using the same setup from Figure $8 \mathrm{a}$. We observe that the difference between unfairness values is negligible. The peaks are a results of randomness present in task acceptance. Therefore, we conclude that unfairness is not effected from the adaption of real-world data to our problem setup.

\subsection{Discussions}

We present two additional experiments that provide insights of our 2-phase assignment model and the F-Aware algorithm. Figure $9 \mathrm{a}$ shows the distribution of workers as a function of the average earning per acceptance. We observe that 800 workers have average values between $2 \$$ and $4 \$$ when F-Aware algorithm is used. Although the most dense areas similar for other approaches as well, we can see that the standard deviation of them is much higher, which is an indicator of unfairness.

Unlike traditional crowdsourcing, in spatial crowdsourcing workers have to physically travel to the source and destination of the task. Therefore, dollar earned per traveled kilometer is a good indicator of the what is the reward of a worker in return of her labor. In this point we would like to remind that, batched progressive offer strategy multicasts the offer to the workers who are most likely to accept it, implicitly workers who are closer to the task.

Figure $9 \mathrm{~b}$ presents dollar earned per kilometer as a function of the coefficient of mean values as in the sensitivity experiments. Solid lines represent different assignment algorithms when the batched progressive offer strategy is used, while dashed lines show the same but when the offers are broadcasted. Recall that the nearest worker assignment is beneficial for capturing the spatial aspect of the assignment problem. In case of broadcasting, represented with dashed lines, we observe the Nearest better than other approaches. Using this approach, workers could make more than 20 cents per kilometer more compared to other approaches. On the other hand, we observe the other three approaches benefit significantly from multicasting. The difference between Nearest and F-Aware decreases from 22 cents to 5 cents when the system prefers to multicast the offers instead of broadcast. Therefore, we could state that F-Aware approach captures the spatial aspect of the problem, with the help of multicasting, as well as allocating each task to Nearest worker.

In summary, our experimental evaluation shows that: 
- F-Aware is efficient: It runs around $10^{7}$ times faster than the MCF algorithm and allocates $98.1 \%$ of the assignable tasks.

- F-Aware is fair: Unfairness metric of the $L A F$ is $2.5 \times$ that of F-Aware and, it maintains its fairness with increasing capacity, and increasing task count.

- Batched progressive offering is useful to limit the assignment ratio, while not sacrificing the wait time. Moreover, it helps to capture the spatial aspect of the problem by helping to provide competitive dollar per kilometer ratio with Nearest worker approach.

\section{Related Work}

We discuss the related work in the areas of crowdsourcing, including spatial crowdsourcing and fairness.

Crowdsourcing. A wide spectrum of crowdsourcing applications are surveyed in [12]. Crowdsourcing is proposed also for technical tasks such as relational query processing [18], [28], [26]. In [20], three crucial aspects of crowdsourcing, namely task design, marketplace dynamics, and worker behavior are analyzed. However, none of these methods from the literature have considered fairness among workers. To the best of our knowledge, this is the first work that applies the concept and findings of fairness from social psychology research to crowdsourcing applications.

Spatial Crowdsourcing. Spatial crowdsourcing requires workers to physically travel to task locations. Earlier work [2], [21], [11] extended crowdsourcing to the physical world, with a variety of applications such as answering queries [18], [28] and serving micro-tasks (such as taking a photo of a monument) [10], [36], [9]. Numerous work has addressed the maximum task assignment problem [21], [11], [31] and its extensions that integrate the reliability of workers [22], [33]. In [10], maximizing the reliability and spatial diversity are considered together. Although these works study task allocation in spatial crowdsourcing, they mostly focus on offline scenarios. In [19], online allocation is performed when only the workers are dynamic. In [36], micro tasks are allocated when both tasks and workers can appear anywhere, anytime. Different from our work, [32] learns the workers' acceptance probability in dynamic tasks static workers setup. Recall, we assume acceptance depends on the hardness level of the tasks and independent from previous acceptances. Unlike these works, in crowdsourced delivery, redundant task allocation is not possible. Similar to our problem, in the context of crowdsensing, there is a trade off among quality of information (QoI), budget and time constraints, which requires multi-objective aware task allocation algorithms as well. A recent work solves this problem with a particle swarm optimization technique that maximizes the aggregated QoI/budget ratio. A delegation mechanism is used, in case the workers cannot finish their allocated task, they may recommend a set of workers from their social network to complete it [15]. This technique serves the same purpose with our 2-phase allocation model. Fairness is not in the scope of any of the aforementioned work.

Fairness in Crowdsourcing. In various application domains, such as networking [27], staff scheduling [14], and resource allocation [4], fairness is considered to be one of the most important constraints. In [8], fairness among customers, but not workers, is considered. Its goal is making sure that the system is fair to the customer when it is not possible to serve all tasks. In [6] unfairness is defined as discrimination against individuals, while in [13], the authors differentiate between various perspectives on fairness and ethics in crowdsourcing. They consider distributional fairness as a subjective measure and discuss ethical implications. In [35], in addition to the cost minimization objective, fair allocation of tasks to heterogeneous workers (workers with different capacities and costs to execute the task) is studied. The central idea of fairness in [35] is to maximize the minimum utility of all bidders (workers). Utility is defined as the number of allocations. They also assume that the set of all tasks is known in advance and workers are predefined. Unlike previous works in spatial crowdsourcing, [25] stresses both the cost incurred by the movement of the worker and the fairness of the assignment among the workers. A sequence of sets of spatial tasks are assigned to crowdsourced workers as they arrive. The one-to-one assignment of tasks to workers is done in mini-batches. In their setup, workers are not assigned to multiple tasks. On the other hand, to provide redundant task allocation they copy the task, and allocate one worker for each copy. Besides utilizing a redundant task allocation strategy, the fairness definition of this work is different from for our scenario. Yet, in the experimental evaluation we included the least allocated worker first, $L A F$, which is inspired from this work. We showed that, according to the state-of-theart fairness definition, $L A F$ is not fair unless all workers are homogeneous.

\section{Conclusion}

In this paper, we created a strategy on allocation of delivery tasks. In this strategy, we use a combined objective of maximum task allocation and fair distribution of tasks to workers. In our 2-phase allocation model, for each task a set of nominees are identified using availabilities of workers. The task is offered to nominees using our batched-progressive offer strategy. Once the candidates for each task are identified, we showed that the problem can be reduced to Minimum Cost Flow(MCF) problem if fairness is not considered. To cope with drawbacks of MCF-based solutions in terms of running time and lack of fairness handling, we introduce our F-Aware algorithm. We then adapt our model to online task allocation and mini-batch task allocation scenarios. Our evaluation showed that $F$-Aware runs around $10^{7} \times$ faster than the TARoptimal solution and assigns $96.9 \%$ of the tasks that can be assigned by it. Moreover, F-Aware assigns 18\% more tasks than random assignment approach and is $2.5 \times$ more fair than the least allocated worker first assignment strategy.

Our experimental evaluation showed that the distributed fairness criteria can be satisfied with no significant changes in task allocation ratios. The proposed approach of fair allocation of tasks can lead to more sustainable crowdsourced delivery platforms. Human perspectives of fairness with quantitative and qualitative surveys, and longterm effects of fair allocation strategies in real crowdsourced 
delivery platforms are among potential future work in this area.

\section{REFERENCES}

[1] J. Adams. Inequity in social exchange. New York Academic Press, 2:267-299, 1965.

[2] F. Alt, A. S. Shirazi, A. Schmidt, U. Kramer, and Z. Nawaz. Location-based crowdsourcing: Extending crowdsourcing to the real world. In Proceedings of Nordic Conf. on HCI: Extending Boundaries, pages 13-22, Reykjavik, Iceland, 2010.

[3] S. Barocas and A. D. Selbst. Big data's disparate impact. 2016.

[4] D. Bertsimas, V. F. Farias, and N. Trichakis. On the efficiencyfairness trade-off. Manage. Sci., 58:2234-2250, Dec. 2012.

[5] L. G. Boiney. When efficient is insufficient: Fairness in decisions affecting a group. Management Science, 41:1523-1537, 1995.

[6] R. M. Borromeo, T. Laurent, M. Toyama, and S. Amer-Yahia. Fairness and transparency in crowdsourcing. In Proc. of the EDBT, pages 466-469, 2017.

[7] A. M. Brawley and C. L. Pury. Work experiences on mturk. Comput. Hum. Behav., 54(C):531-546, Jan. 2016.

[8] A. M. Campbell, D. Vandenbussche, and W. Hermann. Routing for relief efforts. Transportation Science, 42:127-145, May 2008.

[9] Z. Chen et al. gmission: A general spatial crowdsourcing platform. VLDB Conf., 7:1629-1632, Aug. 2014.

[10] P. Cheng, X. Lian, Z. Chen, R. Fu, L. Chen, J. Han, and J. Zhao. Reliable diversity-based spatial crowdsourcing by moving workers. VLDB Conf., 8:1022-1033, June 2015.

[11] D. Deng, C. Shahabi, and U. Demiryurek. Maximizing the number of worker's self-selected tasks in spatial crowdsourcing. In Proc. of ACM SIGSPATIAL, pages 324-333, Orlando, Fl., 2013.

[12] A. Doan, R. Ramakrishnan, and A. Y. Halevy. Crowdsourcing systems on the www. Commun. ACM, 54:86-96, Apr. 2011.

[13] D. Durward, I. Blohm, and J. M. Leimeister. Is there papa in crowd work?: A literature review on ethical dimensions in crowdsourcing. In 2016 Intl IEEE Conf. on Ubiquitous Intelligence Computing, pages 823-832, July 2016.

[14] A. Ernst, H. Jiang, M. Krishnamoorthy, and D. Sier. Staff scheduling and rostering: A review of applications, methods and models. European Journal of Operational Research, 153:3 - 27, 2004.

[15] R. Estrada, R. Mizouni, H. Otrok, A. Ouali, and J. Bentahar. A crowd-sensing framework for allocation of time-constrained and location-based tasks. IEEE Transactions on Services Computing.

[16] R. Faullant, J. Fueller, and K. Hutter. Fair play: perceived fairness in crowdsourcing communities and its behavioral consequences. Academy of Management Proceedings, 2013.

[17] N. Franke, P. Keinz, and K. Klausberger. does this sound like a fair deal?: Antecedents and consequences of fairness expectations in the individuals decision to participate in firm innovation. Organization Science, 24(5):1495-1516, 2013.

[18] M. J. Franklin, D. Kossmann, T. Kraska, S. Ramesh, and R. Xin. Crowddb: Answering queries with crowdsourcing. In ACM Int. Conf. on Management of Data, pages 61-72, Athens, Greece, 2011.

[19] C.-J. Ho and J. W. Vaughan. Online task assignment in crowdsourcing markets. In Proc. of the AAAI Conf. on Artificial Intelligence, pages 45-51, Toronto, Ontario, Canada, 2012.

[20] A. Jain, A. D. Sarma, A. Parameswaran, and J. Widom. Understanding workers, developing effective tasks, and enhancing marketplace dynamics: A study of a large crowdsourcing marketplace. Proc. of the VLDB Endow., 10(7):829-840, Mar. 2017.

[21] L. Kazemi and C. Shahabi. Geocrowd: enabling query answering with spatial crowdsourcing. In Proc. of Int. Conf. on Advances in Geographic Information Systems, pages 189-198, 2012.

[22] L. Kazemi, C. Shahabi, and L. Chen. Geotrucrowd: Trustworthy query answering with spatial crowdsourcing. In Proceedings of ACM SIGSPATIAL, pages 314-323, Orlando, Florida, 2013.

[23] P. Kovcs. Minimum-cost flow algorithms: an experimental evaluation. Optimization Methods and Software, 30:94-127, 2015.

[24] E. Lawler. Combinatorial optimization - networks and matroids. Holt, Rinehart and Winston, New York, 1976.

[25] Q. Liu, T. Abdessalem, H. Wu, Z. Yuan, and S. Bressan. Cost Minimization and Social Fairness for Spatial Crowdsourcing Tasks, pages 3-17. Dallas, TX, USA, 2016.

[26] A. Marcus, E. Wu, D. R. Karger, S. Madden, and R. C. Miller. Demonstration of qurk: A query processor for humanoperators. In ACM Int. Conf. on Management of Data, pages 1315-1318, Athens, Greece, 2011.
[27] W. Ogryczak, M. Pióro, and A. Tomaszewski. Telecommunications network design and max-min optimization problem. Journal of Telecommunications and Information Technology, 3:43-56, 2005.

[28] H. Park, H. Garcia-Molina, R. Pang, N. Polyzotis, A. Parameswaran, and J. Widom. Deco: A system for declarative crowdsourcing. VLDB Conf., 5:1990-1993, Aug. 2012.

[29] J.-F. Rouges and B. Montreuil. Crowdsourcing delivery: New interconnected business models to reinvent delivery. In Int. Physical Internet Conf., Quebec, Canada, May 2014.

[30] S. P. Schappe. Understanding employee job satisfaction: The importance of procedural and distributive justice. Journal of Business and Psychology, 12(4):493-503, 1998.

[31] H. To, C. Shahabi, and L. Kazemi. A server-assigned spatial crowdsourcing framework. ACM Trans. Spatial Algorithms Syst., 1:2:1-2:28, July 2015.

[32] U. Ul Hassan and E. Curry. A multi-armed bandit approach to online spatial task assignment. 2014 IEEE 11th Int. Conf on Ubiquitous Intelligence And Computing, pages 212-219, 2014.

[33] U. Ul Hassan and E. Curry. Efficient task assignment for spatial crowdsourcing: A comb. fractional optimization approach with semi-bandit learning. Exp Systems With Apps., 58:36-56, 2016.

[34] D. Yang, D. Zhang, V. W. Zheng, and Z. Yu. Modeling user activity preference by leveraging user spatial temporal characteristics in lbsns. IEEE Trans. on Systems, Man, and Cybernetics: Systems, 45:129-142, 2015.

[35] Q. Ye, Y. Zhang, and R. Dekker. Fair task allocation in transportation. Omega, pages -, Apr. 2016.

[36] T. Yongxin, S. Jieying, D. Bolin, W. Libin, and C. Lei. Online mobile micro-task allocation in spatial crowdsourcing. In Proc. of ICDE 2016, Helsinki, Finland, May 2016.

[37] M. B. Zafar, I. Valera, M. Gomez Rodriguez, and K. P. Gummadi. Fairness beyond disparate treatment disparate impact: Learning classification without disparate mistreatment. In Proc. of Int. Conf. on WWW, WWW, pages 1171-1180, Republic and Canton of Geneva, Switzerland, 2017.

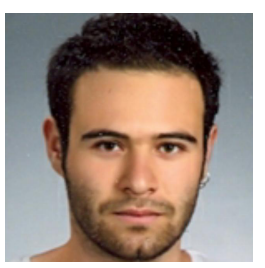

Fuat Basık is a graduate student in the Department of Computer Engineering, Bilkent University, Turkey. He holds a M.Sc. degree in Computer Science from Bilkent University. His research interests are in scalable data integration.

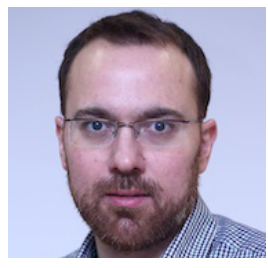

Buğra Gedik is an Associate Professor in the Department of Computer Engineering, Bilkent University, Turkey. He holds a Ph.D. degree in Computer Science from Georgia Institute of Technology. His research interests are in dataintensive distributed systems.

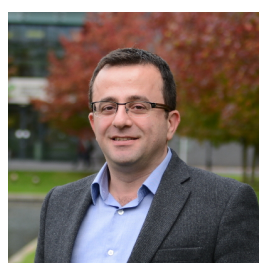

Hakan Ferhatosmanoğlu is a Professor in the Department of Science at the University of Warwick. His research is on scalable data management and analytics for multi-dimensional data. He holds a Ph.D. degree in Computer Science from University of California, Santa Barbara. $\mathrm{He}$ received research career awards from the US Department of Energy, US National Science Foundation, The Science Academy of Turkey, and Alexander von Humboldt Foundation.

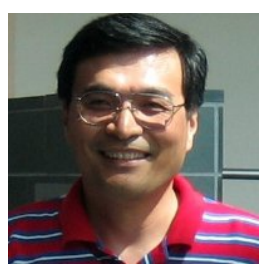

Kun-Lung $\mathbf{W u}$ is a Manager at the IBM T. J. Watson Research Center. His research interests are in big data systems and applications. 